\title{
9. Competition and cooperation in natural resources exploitation: an evolutionary game approach
}

\author{
Gian Italo Bischi, Fabio Lamantia and Lucia \\ Sbragia
}

\section{INTRODUCTION}

Since the pioneering work of Gordon (1954), many bioeconomic models for the description of the commercial exploitation of common property renewable resources, such as fisheries, have stressed the problem known as 'the tragedy of the commons' (Hardin, 1968; see also Clark, 1990). This problem can be basically identified with a prisoner's dilemma (see for example, Mesterton-Gibbons, 1993) because the presence of firms playing their dominant strategy which maximizes their own profit (disregarding competitors' profits) leads to severe depletion of the resource, and consequently to low profits for all. On the other hand if firms cooperate to maximize total profits, then sustainable exploitation is more likely to obtain, which implies higher profits for all in the long run. However, unilateral defection, that is, the decision of an agent to harvest intensively while the other players harvest moderately in order to preserve resources, may lead to very high profits for the defector, and consequently to severe profit loss for the cooperators. This is the essence of the tragedy of the commons, often advanced in order to support the introduction of sanctions against defectors and/or restrictions to open access to common property resources.

Dynamic models based on Cournot oligopoly games have been proposed by Levhari and Mirman (1982) and, more recently, by Szidarovszky and Okuguchi (1998, 2000), to describe commercial fishing. In these models, strategic interaction among players is related not only to the selling price, determined by the total harvesting quantity through a given demand function, but also to a cost externality, since resource stock reductions, as a consequence of players' harvesting, lead to higher unitary fishing costs (see also Bischi and Kopel, 2002). In Szidarovszky and Okuguchi (1998) every player is assumed to decide his/her harvesting activity by solving a profit 
maximization problem, without any concern for the implications of this activity on the depletion of the natural resource. Instead, in Szidarovszky and Okuguchi (2000) it is assumed that the fishermen form a grand coalition (that is, a cooperative venture) and each player determines his/her harvesting activity such that the joint profit of all players is maximized. In both cases, the solution of the optimization problem leads to harvesting functions that depend on fish stocks, the dynamics of which is governed by a biological growth function with an extra mortality term representing harvesting activity.

A discrete time version of the oligopoly model proposed in Szidarovszky and Okuguchi (1998) is given in Bischi and Kopel (2002), where dynamics given perfect foresight (that is, fishermen are assumed to know the fish stock at the time when they solve their maximization problems) are compared with dynamics given limited knowledge of the fish stock and its estimate is obtained by adaptive expectations. In Bischi et al. (2004), discrete time versions of both models (one assuming non-cooperative oligopoly competition as in Szidarovszky and Okuguchi, 1998, and one with total cooperation, where all the players form a unique cooperative venture, so that they behave like a sole owner, as in Szidarovszky and Okuguchi, 2000) are considered with adaptive expectations.

In the model proposed in this chapter, the players (for example, fishermen) have access to a common property resource (for instance, a sea where a given fish stock is available) and sell the harvested resource in the same market. However, in contrast to the above cited literature, both competitors and cooperators are present. In fact, a fraction $s$ of fishermen behaves as cooperators, and form a cooperative venture where each one decides the harvesting quota by maximizing the profit of the coalition, whereas the complementary fraction $(1-s)$ of fishermen behave as competitors (or 'defectors' with respect to cooperative behaviour) each deciding harvesting quotas by maximizing his/her own profit (disregarding competitors' profits). Following the terminology typical of the prisoner's dilemma (see also Sethi and Somanathan, 1996) we call the latter group defectors, because they deviate from the socially optimal attitude of cooperating, and consequently they produce a negative externality on the rest of the community, in terms of potential severe depletion of the common property resource.

As in Szidarovszky and Okuguchi $(1998,2000)$ and Bischi and Kopel (2002), the harvesting of each group depends on strategic interactions related not only to the influence of total supply on the market prices, but also to the dependence of harvesting cost on the available fish stock, whose evolution is governed by biological laws as well as harvesting activities. Moreover, following Sethi and Somanathan (1996), we introduce the possibility that cooperators impose sanctions to punish defectors, and we 
propose an evolutionary mechanism to describe how the population share is updated over time, based on replicator dynamics, that is, on the principle, typical of evolutionary games, that the fraction of agents playing the strategy that earns higher payoffs increases in the next period.

As in Sethi and Somanathan (1996) we assume that, at any time period, the agents decide their harvesting by computing the Nash equilibrium of the game. However, differently from the model proposed in Sethi and Somanathan (1996), where the harvesting behaviour of cooperators and defectors is assumed to be described by general functions that satisfy some formal assumptions, we explicitly derive the profit maximization problem that cooperators and defectors are playing at any time period, founded on given demand and cost functions. This allows us to study, by analytical and numerical methods, the effects of some economic parameters, such as the market price (that is, the parameters that characterize the demand function) or the cost parameters (related to the technology adopted), on the long-run evolution of the resource stock and the population shares between the two groups. In particular, the proposed model allows us to detect which economic parameters may determine not only the extinction of the resource, but also the extinction of a given behaviour.

Another difference between the model studied in this chapter and the one proposed by Sethi and Somanathan, is in the time setting. In fact, the dynamic evolutionary model proposed by Sethi and Somanathan evolves in continuous time. This means that they assume that at every time instant a player can change his behaviour from cooperator to defector (or vice versa) according to the instantaneous profits. We consider this a strong assumption, because if a player decides upon a given behaviour, he will maintain that kind of behaviour for a given time period. In other words, we assume that he cannot change his mind just after his decision, as a minimum time lag is necessary to decide a change of behaviour, on the basis of observed profits. Such a time lag, which constitutes unitary time in our discrete time setting, may be assumed to be one week, or one month, according to the economic and social framework considered.

The chapter is organized as follows. In Section 2 we propose a static game where a population of profit maximizing agents decide the quantities to harvest on the basis of two different behaviours: a fraction of players form a coalition, each of them trying to maximize the overall profit, whereas the complementary fraction behave as 'selfish' profit maximizers. The reaction functions are obtained and the unique Nash equilibrium of the game is computed. In Section 3 a growth equation, governing the dynamics of the resource stock with harvesting, is introduced, the harvesting being decided by the two groups by choosing the Nash equilibrium quantities according to the game analyzed in Section 2. In this section the 
population share between the two kinds of agents is assumed to be a parameter, so the existence and stability of the steady states are studied as well as how these are influenced by population share. In Section 4 we introduce an evolutionary mechanism that, at each time period, describes how the population share is updated, based on replicator dynamics, and we study the problems of existence and stability of the equilibria. In particular a distinction is introduced between boundary equilibria, where all the players behave as cooperators or as defectors, and inner equilibria, where cooperators and defectors coexist in the long run. A short discussion of the results, a description of open problems and possible further developments are given in Section 5.

\section{THE STATIC GAME}

Let us assume that a population of $n$ agents harvests from a common property renewable resource stock, and sells the harvested resource at a price $p$ determined by the total harvested quantity according to a given demand function. For example, we may imagine that the agents are fishermen who harvest fish from a sea where a given fish stock $X$ is present. However, similar considerations may be applied to the harvesting of different renewable resources, such as forests or others. The agents decide the quantities to harvest on the basis of profit maximization problems. However, we assume that a fraction $s$ of them, denoted as 'cooperators' form a coalition (a cooperative venture) and consequently each of them tries to maximize the overall profit of the coalition, whereas the remaining fraction $(1-s)$ behave as 'selfish' profit maximizers, and are denoted as 'defectors' (with respect to the socially more desirable cooperative behaviour).

In this section we consider $X$ and $s$ as fixed parameters, ${ }^{1}$ with $X>0$ and $0 \leq s \leq 1$. Let $x_{c}^{i}$ be the quantity harvested by the cooperator $i, i=1, \ldots, n s$, and let $x_{d}^{i}$ be the quantity harvested by defector $i, i=1, \ldots, n(1-s)$. Then the total fish supplied and sold in the market is

$$
H=\sum_{i=1}^{n s} x_{c}^{i}+\sum_{i=1}^{n(1-s)} x_{d}^{i}
$$

We assume that the selling price $p$ is determined by the linear demand function

$$
p=a-b H
$$

where $a$ and $b$ are positive constants, and the cost function of player $i$ for harvesting a quantity $x$ when a fish stock $X$ is present is given by 


$$
C(x, X)=\gamma \frac{x^{2}}{X}
$$

This cost function can be derived from a Cobb-Douglas-type 'production function' with fishing effort (labour) and fish biomass (capital) as production inputs (see Clark, 1990; Szidarovszky and Okuguchi, 1998). It captures the fact that it is easier and less expensive to catch fish if the fish population is large.

Following Sethi and Somanathan (1996) we shall also consider an extra cost due to the presence of social norms that are intended to punish fishermen who behave as defectors, that is, self-interested profit maximizers without any concern for the social optimum. As in Sethi and Somanathan (1996) we assume that cooperators are entrusted to punish defectors by applying sanctions. This may be done directly by exerting a direct punishment, such as social disapproval damage or physical damage or destruction of equipment, as observed in less developed societies, or by alerting authorities so that they can impose sanctions according to the laws in force. Such punishment is costly for the defectors, the cost being $n s \xi$, where $\xi$ is the amount of the sanction and $n s$ represents the probability that a defector is notified by a cooperator. However, in general, this kind of punishment is also costly for the cooperators, the cost being proportional to the number of defectors. We shall represent by $n(1-s) \psi$ this extra cost for cooperators (of course $\xi>\psi$, and we shall often consider $\psi=0$ in the following). All in all, the profit of $i$-th cooperator is

$$
\pi_{c}^{i}=x_{c}^{i}(a-b H)-\gamma_{c} \frac{\left(x_{c}^{i}\right)^{2}}{X}-n(1-s) \psi
$$

where $\gamma_{c}$ is the fishing technology coefficient of cooperators and $n(1-s) \psi$ represents the cost that cooperators have to face in order to punish defectors, and the profit of $i$-th defector is

$$
\pi_{d}^{i}=\mathrm{x}_{d}^{i}(a-b H)-\gamma_{d} \frac{\left(x_{d}^{i}\right)^{2}}{X}-n s \xi
$$

where $\gamma_{d}$ is the fishing technology coefficient of defectors and $n s \xi$ represents the punishment that defectors have to bear for causing the negative externality in the community.

Each cooperator determines $x_{c}^{i}$ by solving the optimization problem

$$
\max _{x_{c}^{i}} \pi^{V}=\max _{x_{c}^{i}} \sum_{i=1}^{n s} \pi_{c}^{i}
$$

where $\pi^{V}$, which is a concave function in the variables $x_{c}^{i}$, denotes the total profit of the cooperative venture. Assuming interior optimum, the firstorder conditions give a system of linear equations in the unknowns $x_{c}^{i}$ 


$$
\frac{\partial \pi^{V}}{\partial x_{c}^{i}}=a-2 b \sum_{k=1}^{n s} x_{c}^{k}-b \sum_{k=1}^{n(1-s)} x_{d}^{k}-\frac{2 \gamma_{c}}{X} x_{c}^{i}=0
$$

Each defector determines $x_{d}^{i}$ by solving the optimization problem

$$
\max _{x_{d}^{i}} \pi_{d}^{i}
$$

Assuming, again, interior optimum, the first-order conditions give a system of linear equations in the unknowns $x_{d}^{i}$

$$
\frac{\partial \pi_{d}^{i}}{\partial x_{d}^{i}}=a-b \sum_{k=1}^{n s} x_{c}^{k}-b \sum_{k=1}^{n(1-s)} x_{d}^{k}-b x_{d}^{i}-\frac{2 \gamma_{d}}{X} x_{d}^{i}=0
$$

The equations (9.6) and (9.8) give a linear system of $n$ equations with $n$ unknowns. However, it is straightforward to see that any cooperator faces the same optimization problem, and analogously for the defectors. In fact, if we denote by $x_{c}^{T O T}=\sum_{k=1}^{s n} x_{c}^{k}$ the total harvest of the cooperators and by $x_{d}^{T O T}=\sum_{k=1}^{(1-s) n} x_{d}^{k}$ the total harvest of the defectors, from (9.6) we get

$$
x_{c}^{i}=\frac{X}{2 \gamma_{c}}\left(a-2 b x_{c}^{T O T}-b x_{d}^{T O T}\right) \quad \forall i=1, \ldots, s n
$$

and from (9.8) we get

$$
x_{d}^{i}=\frac{X}{b X+2 \gamma_{d}}\left(\mathrm{a}-\mathrm{b}\left(x_{c}^{T O T}+x_{d}^{T O T}\right)\right) \quad \forall i=1, \ldots,(1-s) n
$$

So, denoting the optimal harvesting decision of each cooperator by $x_{c}$, and the optimal harvesting decision of each defector by $x_{d}$, these quantities are obtained by solving the two linear equations

$$
\begin{gathered}
a-2\left(b s n+\frac{\gamma_{c}}{X}\right) x_{c}-b(1-s) n x_{d}=0 \\
a-b \operatorname{sn} x_{c}-\left[b(1+(1-s) n)+\frac{2 \gamma_{d}}{X}\right] x_{d}=0
\end{gathered}
$$

from which two linear reaction functions are obtained

$$
\begin{gathered}
x_{c}=h_{c}\left(x_{d}\right)=\frac{a X}{2\left(b s n X+\gamma_{c}\right)}-\frac{b(1-s) n X}{2\left(b s n X+\gamma_{c}\right)} x_{d} \\
x_{d}=h_{d}\left(x_{c}\right)=\frac{a X}{b(1+(1-s) n) X+2 \gamma_{d}}-\frac{b s n X}{b(1+(1-s) n) X+2 \gamma_{d}} x_{c}
\end{gathered}
$$


These reaction functions allow one to compute, respectively, the optimal harvesting decision of a 'representative cooperator', given the harvesting decision of a representative defector, and the optimal harvesting decision of a 'representative defector', given the harvesting decision of a representative cooperator. These two reaction functions always intersect at a unique point $\left(x_{d}^{*}, x_{c}^{*}\right)$ whose coordinates are functions of fish stock $X$ and the fraction of cooperators $s$, as well as of the cost parameters $\gamma_{c}, \gamma_{d}$ and the market price parameters $a$ and $b$, according to the expressions given in the Appendix in (9.29) and (9.30). The properties of the harvesting strategies $x_{d}^{*}$ and $x_{c}^{*}$, of defectors and cooperators respectively, at the Nash equilibrium are given in the following Proposition

Proposition 1. A unique Nash Equilibrium $\left(x_{d}^{*}, x_{c}^{*}\right)$ exists, with $x_{d}^{*}>0$ and $x_{c}^{*}>0$, located at the intersection of the reaction curves (9.9), such that:

(i) For each $s \in[0,1]$ both $x_{d}^{*}(0, s)$ and $x_{c}^{*}(0, s)$ vanish for $X=0$, are positive for $X>0$ and at $X=0$ have slopes given by

$$
{\frac{\partial x_{d}^{*}(X, s)}{\partial X} \mid X=0}_{\mid X \gamma_{d}} ; \quad \frac{a x_{c}^{*}(X, s)}{\partial X \quad \mid X=0}=\frac{a}{2 \gamma_{c}}
$$

(ii) Both $x_{d}^{*}(0, s)$ and $x_{c}^{*}(0, s)$ tend to saturate as $X \rightarrow+\infty$, at the values

$$
x_{d}^{*}(+\infty, s)=\frac{a}{b[(1-s) n+2]} \quad \text { and } x_{c}^{*}(+\infty, s)=\frac{a}{b \sin [(1-s) n+2]}
$$

respectively;

(iii) The gap between $x_{d}^{*}$ and $x_{c}^{*}$, for large values of the fish stock, increases with increasing prices and with the number ns of cooperators, being

$$
x_{d}^{*}(+\infty, s)-x_{c}^{*}(+\infty, s)=\frac{a}{b[(1-s) n+2]}\left(1-\frac{1}{s n}\right)
$$

(iv) The total harvesting at the Nash equilibrium, given by

$$
H^{*}(X, s)=n\left[s x_{c}^{*}(X, s)+(1-s) x_{d}^{*}(X, s)\right]
$$

is an increasing and concave function with respect to $X$, such that, $H^{*}(0, s)=0$,

$$
{\frac{\partial H^{*}(X, s)}{\partial X} \mid X=0}=\frac{n a}{2}\left(\frac{s}{\gamma_{c}}+\frac{1-s}{\gamma_{d}}\right)
$$

and for $X \rightarrow+\infty$ it saturates at the value 


$$
H^{*}(+\infty, s)=\frac{a}{b}\left(1-\frac{1}{2+(1-s) n}\right)
$$

A proof of this proposition is outlined in the Appendix.

Typical graphs of $x_{d}^{*}$ and $x_{c}^{*}$, as functions of $X$, are shown in Figure 9.1a, and a typical graph of $H^{*}$ is shown in Figure 9.1b. The properties of the harvesting function $H^{*}$ are similar to the ones assumed by Sethi and Somanathan (1996), but in our case the harvesting function is micro-

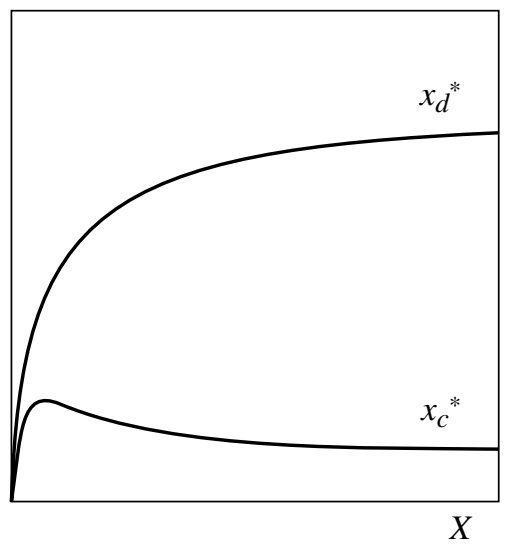

(a)

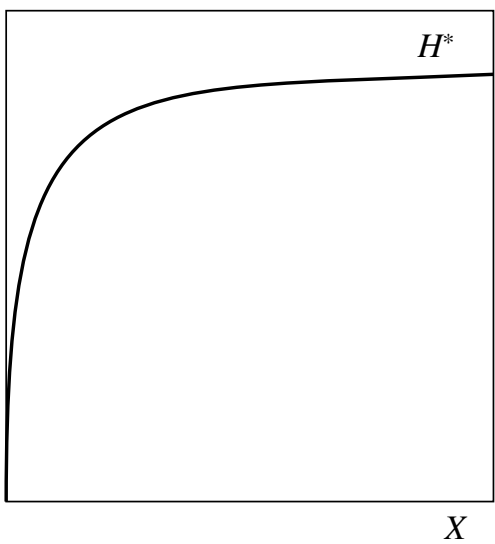

(b)

Notes:

(a) Harvesting of defectors $x_{d}^{*}$, and cooperators, $x_{c}^{*}$. (b) Total harvesting $H^{*}$

Figure 9.1 Typical graphs of harvesting quantities at the Nash equilibrium depending on the available resource stock $\mathrm{X}$

founded, that is, it is derived from an explicit underlying optimization problem. This implies that the effects of the economic and biological parameters can be explicitly studied. First of all we notice that, for $s n \geq 2$ (that is, if at least two cooperators exist) then $x_{c}^{*}<x_{d}^{*}$ for large values of fish stock, whereas for small values of $X$ the comparison between $x_{d}^{*}$ and $x_{c}^{*}$ depends on the respective cost parameters. It is also worth noticing that both $x_{d}^{*}$ and $x_{c}^{*}$ increase if the selling price increases, that is, $a$ increases and/or $b$ decreases in the demand function (9.1).

Notice also that $H^{*}<a / b$ for each $X>0$, so that prices are always positive. Moreover, if $s$ decreases, that is, the number of defectors increase, then $H^{*}(+\infty, s)$ increases, that is, as expected, in the presence of an abundant 
resource the total harvest is greater if the number of defectors increases. In the limiting case $s=0$ (all defectors) we have $H^{*}(+\infty, 0)=a / b(1-1 / 2+n)$, and in the opposite limiting case $s=1$ (all cooperators, equivalent to the case of a sole owner) we get $H^{*}(+\infty, 1)=a / 2 b$, the monopolist optimum.

These results given in Proposition 1 allow us to compute, at the Nash equilibrium, the profit of a representative defector and that of a representative cooperator, given by

$$
\begin{aligned}
& \pi_{d}^{*}=x_{d}^{*}\left(a-b H^{*}\right)-\gamma_{d} \frac{\left(x_{d}^{*}\right)^{2}}{X}-n s \xi \text { and } \\
& \pi_{c}^{*}=x_{c}^{*}\left(a-b H^{*}\right)-\gamma_{c} \frac{\left(x_{c}^{*}\right)^{2}}{X}-n(1-s) \psi
\end{aligned}
$$

respectively. Plugging the expression of $x_{c}^{*}, x_{d}^{*}$ and $H^{*}$ into $(9.11), \pi_{d}^{*}$ and $x_{c}^{*}$, can be rewritten as:

$$
\begin{gathered}
\pi_{d}^{*}= \\
\frac{a^{2} X\left(b n s X+2 \gamma_{c}\right)^{2}\left(b X+\gamma_{d}\right)}{\left[b X(b n(-n(1-s)-2)) s X-2 b X(n(1-s)+1) \gamma_{c}-4\left(b n s X+\gamma_{c}\right) \gamma_{d}\right]^{2}} \\
-n s \xi
\end{gathered}
$$

and

$$
\begin{gathered}
\pi_{c}^{*}= \\
\frac{a^{2} X\left(b n s X+\gamma_{c}\right)\left(b X+2 \gamma_{d}\right)^{2}}{\left[b X(b n(-n(1-s)-2)) s X-2 b X(n(1-s)+1) \gamma_{c}-4\left(b n s X+\gamma_{c}\right) \gamma_{d}\right]^{2}} \\
-(1-s) n \psi
\end{gathered}
$$

respectively. These expressions show that, at the Nash equilibrium, the profits of defectors (cooperators) are positive provided that the applied sanctions (the costs to apply sanctions) are not too heavy. For example, under the assumptions $\psi=0$ and $\xi>0$, which we shall often consider in the following, we have $\pi_{c}^{*}$ always positive and $\pi_{d}^{*}$ positive or negative according to the sanctions applied and to the number of cooperators.

\section{THE ONE-DIMENSIONAL DYNAMICS WITH FIXED $s$}

We now regard $X$ as a dynamic variable and $s$ as a parameter, that is, we consider the time evolution of the resource stock $X(t)$, that depends on its natural growth function and on the harvesting activity, and we assume a fixed division of fishermen population between cooperators and defectors. 
Let $X(t)$ denote the fish stock at time period $t$. We consider the following discrete time equation to describe the time evolution of the fish stock

$$
X(t+1)=F(X(t))=X(t)(1+\alpha-\beta X(t))-H^{*}(X(t), s)
$$

that is, we assume that in the absence of any harvesting the stock of the fish population in period $t$ is determined by the discrete time logistic equation, ${ }^{2}$ with $\alpha$ and $\beta$ biological parameters that characterize the fish population we are considering and the environment where it lives: $\propto$ is the intrinsic growth rate and $\alpha / \beta$ the carrying capacity, that is, the positive equilibrium value of the unharvested resource population, since for $H^{*}=0$ and $X=\alpha / \beta(9.14)$ gives $X(t+1)=X(t)$. We also assume that the fish stock is harvested according to the Nash equilibrium of the static game described in the previous section. Under these assumptions, and by imposing in (9.14) the equilibrium condition $X(t+1)=X(t)$, we get that the steady states of the model with harvesting are the non-negative solutions of the equation

$$
X(\alpha-\beta X)=H^{*}\left(X ; s, \gamma_{c}, \gamma_{d}, a, b, n\right)
$$

So, for any given value of $s$, the equilibria are located at the non-negative intersections between a parabola and the concave function (9.10) (see Figure 9.2) and the following Proposition holds (the three situations denoted by (a), (b) and (c) in Proposition 2 are represented, respectively, by the curves $a, b$ and $c$ of Figure 9.2).

Proposition 2. The point $X_{0}=0$ (extinction of the resource) is an equilibrium point for each set of parameters. Concerning the existence of positive equilibria, we can distinguish the following three different situations:

(a) A unique positive equilibrium exists, say $X_{1}$, with $0<X_{1}<\alpha / \beta$. A sufficient condition for this is

$$
\frac{n a}{2}\left(\frac{s}{\gamma_{c}}+\frac{1-s}{\gamma_{d}}\right)<\alpha
$$

(b) Two positive equilibria exist, say $X_{2}$ and $X_{1}$, such that $0<X_{2}<\alpha / 2 \beta<$ $X_{1}<\alpha / \beta$. A sufficient condition for this is

$$
\frac{n a}{2}\left(\frac{s}{\gamma_{c}}+\frac{1-s}{\gamma_{d}}\right)>\alpha \text { and } \frac{a}{b}\left(1-\frac{1}{2+(1-s) n}\right)<\frac{\alpha^{2}}{4 \beta}
$$

(c) No positive equilibria exist. A necessary condition for this is

$$
\frac{n a}{2}\left(\frac{s}{\gamma_{c}}+\frac{1-s}{\gamma_{d}}\right)>\alpha \text { and } \frac{a}{b}\left(1-\frac{1}{2+(1-s) n}\right)>\frac{\alpha^{2}}{4 \beta}
$$




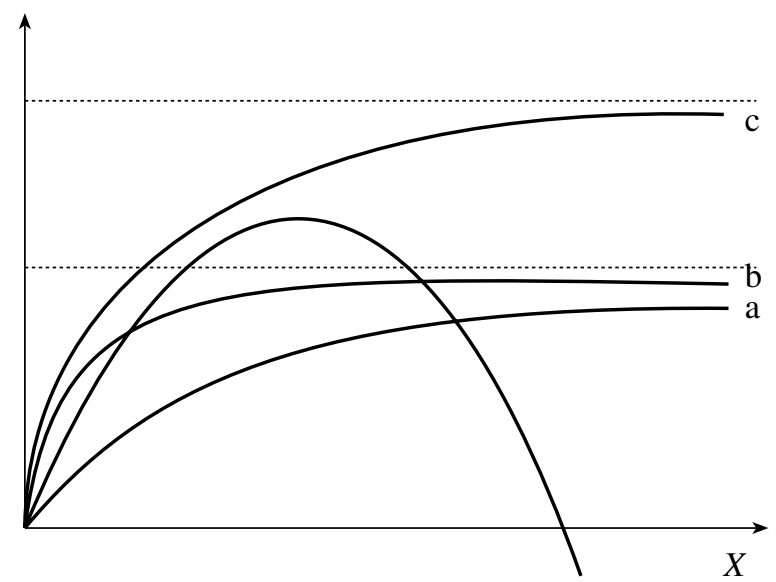

Notes:

The curves denoted by a, b, c can be obtained, respectively, by decreasing values of $s$ or increasing values of $a$ or decreasing values of $b$.

Figure 9.2 Qualitative graphical representation of equation (9.16) with fixed values of the parameters $\alpha$ and $\beta$ and three different functions $H^{*}$

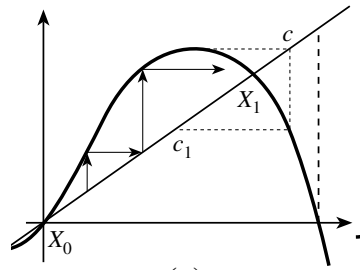

(a)

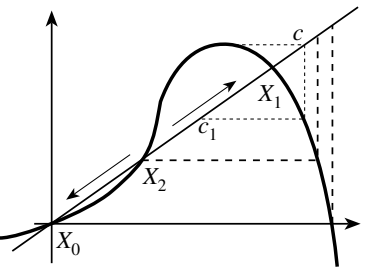

(b)

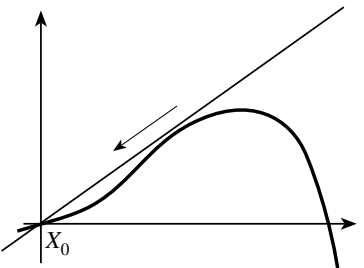

(c)

Notes:

(a) One positive equilibrium; (b) Two positive equilibria, (c) No positive equilibria.

Figure 9.3 Qualitative representation of the function $F$, that governs the one-dimensional dynamics of the resource stock $X$ according to (9.15), in the three different cases represented in Figure 9.2

A proof of this proposition is outlined in the Appendix.

Proposition 2 only concerns existence of equilibria. We now give conditions for their stability and local bifurcations that cause stability switches as well as creation or destruction of positive equilibria. We start from the case of 
a unique positive equilibrium. If (9.16) holds, then $D F(0)>1$, so the fixed point $X_{0}=0$ is unstable (see Figure 9.3a). This means that if (9.16) is satisfied, that is, the number of fishermen is not too high, prices are not too high nor cost parameters too small, then even if the resource stock is reduced to an arbitrary small positive value by some exogenous shock, the endogenous dynamics of the system is such that it spontaneously evolves to viable levels of the resource stock, close to $X_{1}$. The unique positive equilibrium $X_{1}$ may be stable, that is, $\left|D F\left(X_{1}\right)\right|<1$, or unstable, with $D F\left(X_{1}\right)<$ -1 . In the latter case, which occurs with high values of the parameter $\alpha$, a more complex bounded attractor, which may be periodic or chaotic, may exist around $X_{1}$. The bounded attracting set is confined inside the trapping set $I=\left[c_{1}, c\right]$ where $c$ is the maximum value of the function $F$ and $c_{1}=F(c)$. In any case, the basin of the bounded attractor is bounded by the unstable fixed point $X_{0}=0$ and its rank-1 pre-image $X_{0-1}$, that is,

$$
\mathrm{B}=\left(0, X_{0-1}\right)
$$

where $X_{0-1}$ is the positive solution of the equation

$$
1+\alpha-\beta X=\frac{H^{*}(X)}{X}
$$

It is straightforward to see that under the assumption (9.16) the equation (9.19) has a unique positive solution $X_{0-1}$ such that $X_{1}<X_{0-1}<(1+\alpha) / \beta$. An initial condition with $X(0)>X_{0-1}$ is mapped by the iterated function $F$ to a negative value, so we consider as unfeasible such kinds of trajectory. ${ }^{3}$

It is worth noticing that the equilibrium value $X_{1}$ is influenced by the value of $s$. In fact, if $s$ is increased, then the asymptote of $H^{*}$ moves downwards, and this implies that $X_{1}$ increases with $s$, that is, $X_{1}(s)$ is an increasing function. The intuition behind this is clear: more cooperators imply a higher resource stock at the long-run equilibrium due to a more conservative (or sustainable) resource exploitation.

If the aggregate parameter at the left-hand side of (9.16) is increased, that is, the number of fishermen and/or prices become higher compared with intrinsic growth of the fish species in the environment considered, and/or the fishing costs are lowered by using more sophisticated technologies, for

$$
n a\left(\frac{s}{\gamma_{c}}+\frac{1-s}{\gamma_{d}}\right)=2 \alpha
$$

we have $D F(0)=1$ and if $n a /\left(s / \gamma_{c}+(1-s) / \gamma_{d}\right)$ is further increased (or $\alpha$ decreased) then a transcritical (or stability exchange) bifurcation occurs after which the equilibrium $X_{0}=0$ becomes stable, that is, $-1<D F(0)<1$, 
and a second fixed point $X_{2}$ enters the positive orthant, thus giving the situation (b). The new positive equilibrium $X_{2}$ is unstable, being $D F\left(X_{2}\right)>1$, and it belongs to the boundary which separates the basin of the stable equilibrium $X_{0}=0$ from the basin of the positive attractor. So, in this situation $X_{2}$ constitutes a threshold population level such that if the current population $X(t)$ falls below $X_{2}$ then the resource stock will spontaneously evolve towards extinction. It is worth noticing that if $s$ increases, so that the asymptote of $H^{*}$ moves downwards, then threshold value $X_{2}$ moves to the left, that is, it is a decreasing function of $s$. This means that more cooperators imply not only a higher resource stock at the long-run equilibrium $X_{1}$, but also an enlargement of its basin of attraction.

As usual with non-invertible maps, all the rank-1 pre-images of $X_{2}$ belong to basin boundaries, so the basin of the positive attractor is now given by

$$
\mathrm{B}=\left(X_{2}, X_{2-1}\right),
$$

$X_{2-1}$ being the greater solution of the equation $F(X)=X_{2}$. The set of positive initial conditions which asymptotically converge to $X_{0}=0$, and give rise to extinction in the long run, is formed by the union of two disjoint intervals

$$
\mathrm{BP}(0)=\left(0, X_{2}\right) \cup\left(X_{2-1}, X_{0-1}\right)
$$

whereas the initial condition $X(0)>X_{0-1}$ generates unfeasible trajectories.

When two positive equilibria exist, the dynamic scenario is the one described above, and represented in Figure 9.3b. With given values of the biological parameters $\alpha$ and $\beta$, so that the parabola in Figure 9.2 is fixed, if the other parameters are varied with the consequence that the asymptote of $H^{*}$ moves upwards, the two positive equilibria become closer and closer, so that the basin of $X_{0}$ enlarges and, therefore, the basin of the viable equilibrium $X_{1}$ shrinks. This can be obtained, for example, by increasing prices (that is, increasing $a / b$ ) or with decreasing values of $s$, that is, by increasing the number of defectors. We are particularly interested in the latter effect: decreasing values of $s$ imply less robustness of the viable equilibrium with respect to exogenous shocks. Of course, a study of the effects of the parameter $\beta$ may also be interesting, as a higher value of $\beta$ may be interpreted as the effect of a damaged environment, due to pollution or other factors.

Finally, the situation $(c)$, where the extinction equilibrium $X_{0}=0$ is the unique steady state, may be obtained as the final effect of increasing $H^{*}(+\infty, s)$. The transition from a dynamic scenario characterized by two positive steady states to one with no positive steady states occurs via a fold 
(or tangent) bifurcation, due to a progressive decrease of $X_{1}$, increase of $X_{2}$ (so that the basin B becomes smaller and smaller) until they merge with $D F\left(X_{1}\right)=D F\left(X_{2}\right)=1$, and then disappear. It is trivial to prove that when $X_{0}$ is the unique equilibrium, then for every initial condition the system evolves towards extinction (see Figure 9.3c). Once more, we remark that a sequence of bifurcations such as that described above may occur for increasing prices, decreasing costs or decreasing values of $s$, that is, by increasing the number of defectors.

\section{THE TWO-DIMENSIONAL MODEL WITH EVOLUTIONARY DYNAMICS OF $s$}

We now relax the assumption of a fixed population share between cooperators and defectors, and we introduce an evolutionary mechanism that, at each time period, describes how the population share is updated. In the spirit of evolutionary games, we assume that the fraction of agents playing a strategy that, with respect to the other strategies, earns higher payoffs, increases in the next period. In our case, the payoffs associated with the two available strategies of cooperation and defection are the profits $\pi_{c}^{*}(t)$ and $\pi_{d}^{*}(t)$ respectively, that, according to (9.13) and (9.12), depend on the current population share $s(t)$ as well as on the current resource stock $X(t)$. Since, as argued in the previous section, the dynamics of $X(t)$ are influenced by $s(t)$, this will give rise to a two-dimensional non-linear dynamical system with dynamic variables $X(t)$ and $s(t)$, the study of which may give us information on the long-run evolution of the system. For example, we may ask not only if the resource stock will survive or become extinct in the long run, but we may also ask if some behaviour (cooperation or defection) will survive or become extinct as the system evolves. Since, under the reasonable assumption $\gamma_{c}=\gamma_{d}$ (that means that the two groups adopt the same technology) the strategy chosen by defectors is dominant if no sanctions are applied, the assumption $\xi>\psi \geq 0$ will be crucial in order to obtain stable equilibria with a non-vanishing fraction $s$ of cooperators.

\subsection{Replicator Dynamics}

The simplest (and more frequently used) model proposed in the literature which gives an evolutionary pressure in favour of groups obtaining the highest payoffs is that of replicator dynamics (Taylor and Jonker, 1978; see also Vega-Redondo, 1996, chapter 3; Hofbauer and Sigmund, 1998, chapter 7; Weibull, 1995, chapter 3). The discrete time replicator dynamics for the fraction of cooperators can be written as 


$$
s(t+1)=s(t) \frac{\pi_{c}^{*}(t)}{\bar{\pi}}
$$

where

$$
\bar{\pi}(t)=s \pi_{c}^{*}(t)+(1-s) \pi_{d}^{*}(t)
$$

represents the average profit observed at time $t$. So, (9.23) states that $s(t+1)$ will be greater than $s(t)$ if $\pi_{c}^{*}(t)>\bar{\pi}(t)$ whereas $s(t)$ will decrease if $\pi_{c}^{*}(t)<\bar{\pi}(t)$. As $\pi_{c}^{*}(t)>\bar{\pi}(t)$ if and only if $\pi_{c}^{*}(t)>\pi_{d}^{*}(t)$, it follows that the population share related to the better performing strategy at time period $t$ increases in the next period.

Both $\pi_{c}^{*}(t)$ and $\bar{\pi}(t)$ depend on $s(t)$, as well as $X(t)$ so the difference equations (9.14) and (9.23) define a two-dimensional discrete dynamical system. Starting from a given initial biomass $X(0)$ and a given initial population share $s(0)$, the recurrences (9.14) and (9.23) allow one to obtain $X(t)$ and $s(t)$ for each $t \geq 0$, from which the corresponding values of $x_{c}^{*}(t), x_{d}^{*}(t), \pi_{c}^{*}(t)$, $\pi_{d}^{*}(t), H^{*}(t)$ can be obtained.

It is interesting to study whether the dynamic variables $X(t)$ and $s(t)$ converge to a given steady state in the long run, that is, as $t \rightarrow+\infty$, or if they exhibit some more complex time pattern.

\subsection{Qualitative Study of the Two-Dimensional Dynamical System}

The time evolution of the discrete time two-dimensional dynamical system in the dynamic variables $X$ and $s$ is obtained by the iteration of a map of the plane $T:(X(t), s(t)) \rightarrow(X(t+1), s(t+1))$ defined by

$$
\begin{gathered}
X(t+1)=X(t)(1+\alpha-\beta X(t))-H^{*}(X(t), s(t)) \\
s(t+1)=s(t) \frac{\pi_{c}^{*}(X(t), s(t))}{s \pi_{c}^{*}(X(t), s(t))+(1-s) \pi_{d}^{*}(X(t), s(t))}
\end{gathered}
$$

where $H^{*}(X, s)$ is given by (9.10), with $x_{c}^{*}$ and $x_{d}^{*}$ defined in (9.30) and (9.29) respectively, and $\pi_{c}^{*}(t), \pi_{d}^{*}(t)$ are given in (9.13) and (9.12) respectively.

It is straightforward to see that if $\pi_{c}^{*}(t)>0$ and $\pi_{d}^{*}(t)>0$ then $s(t) \in(0,1)$ implies $s(t+1) \in(0,1)$ as well. However, negative profits may arise if the parameters $\xi$ and/or $\psi$ are positive. As the influence of these parameters is always related to the difference $\xi-\psi$, without loss of generality we shall assume $\psi=0$, and whenever $\pi_{d}^{*}(t)<0$ we shall assume $s(t+1)=1$ (instead of the meaningless $s(t+1)>1$, as obtained by simply applying (9.25)). 


\subsection{Two Benchmark Cases}

It is important to notice that if $s(t)=0$ then $s(t+1)=0$ for each $t \geq 0$, and if $s(t)=1$ then $s(t+1)=1$ for each $t \geq 0$, that is, the two boundary lines $s=0$ and $s=1$ are trapping lines, on which the dynamics are governed by one-dimensional unimodal maps, given by the restrictions of the two-dimensional map (9.25) to them. These two cases correspond to particular benchmark cases, where we have all cooperators and all defectors respectively, that is, the cases considered in Szidarovszky and Okuguchi (1998) and Szidarovszky and Okuguchi (2000) respectively (see also Bischi et al., 2003). The properties of these one-dimensional dynamical systems can easily be obtained on the basis of the results of Section 3. For example, the dynamics along the invariant edge $s=0$, where all players are defectors, is governed by the one-dimensional map (9.14) with

$$
H^{*}(X, 0)=n x_{d}^{*}(X, 0)=\frac{n a X}{b(n+1) X+2 \gamma_{d}}
$$

So the steady states are $X=0$ and the solutions (if any) of the equation

$$
b \beta(n+1) X^{2}+\left(2 \beta \gamma_{d}-\alpha b(n+1)\right) X+n a-\alpha \gamma_{d}=0
$$

The analysis is the same as in Szidarovszky and Okuguchi (1998) or in Bischi et al. (2003).

On the other invariant edge $s=1$, where all players are cooperators, the dynamics are governed by $(9.14)$ with

$$
H^{*}(X, 1)=n x_{c}^{*}(X, 1)=\frac{n a X}{2\left(b n X+\gamma_{c}\right)}
$$

So the fixed points are $X=0$ and the solutions (if any) of the equation

$$
2 b \beta n X^{2}+2\left(\beta \gamma_{c}-\alpha b n\right) X+n a-2 \alpha \gamma_{c}=0
$$

The analysis is the same as in Szidarovszky and Okuguchi (2000) or in Bischi et al. (2003).

\subsection{Steady States}

As usual, the starting point for the qualitative analysis of a non-linear dynamical system is the localization of the steady states and the study of their local stability. The steady states of the two-dimensional dynamical system (9.25) are the fixed points of the map $T$, solutions of the system $T(X, s)=(X, s)$. It is straightforward to see that two corner equilibria always 
exist, given by $E_{0}=(0,0)$ and $E_{1}=(0,1)$, characterized by extinction of the resource. Other boundary equilibria may exist along the invariant lines $s=$ 0 and $s=1$, given by the solutions, if any, of (9.26) and (9.27) respectively. If two equilibria with positive fish stocks exist both on the invariant edge $s$ $=0$ and on invariant edge $s=1$, say $X_{2}(0), X_{1}(0)$ and $X_{2}(1), X_{1}(1)$, respectively, then, on the basis of the arguments of Section 3, the following relation must hold: $X_{2}(1)<X_{2}(0)<X_{1}(0)<X_{1}(1)$. A necessary condition for the existence of two positive equilibria along $s=0$ is that two positive equilibria exist along $s=1$. However, it may happen that two positive equilibria exist along $s=1$ and no positive equilibria exist along $s=0$. Of course, sufficient conditions for the existence of two positive boundary equilibria along the invariant edges are obtained from (9.17) with $s=0$ and $s=1$ respectively.

Instead, if (9.16) is satisfied for $s=1$, that is, $n a<2 \alpha \gamma_{c}$, then a unique equilibrium exists along the edge with only cooperators, and the same holds on the other invariant edge if $n a<2 \alpha \gamma_{d}$. These two conditions are equivalent under the reasonable assumption $\gamma_{c}=\gamma_{d}$. However, even when a unique equilibrium exists, the inequality $X_{1}(0)<X_{1}(1)$ holds, that is, the long-run equilibrium under sustainable fishing is characterized by higher values of resource stock in the limiting case of all cooperators than in the opposite limiting case of all defectors.

The stability of these equilibria with respect to the one-dimensional dynamics trapped inside the invariant edges can easily be deduced from the discussion on the one-dimensional dynamics given in Section 3 applied to the particular benchmark cases $s=0$ and $s=1$. However, we are now mainly interested in the stability with respect to perturbations transverse to the invariant edges, that is, what happens if a few defectors appear starting from a situation with all cooperators, or what happens if a few cooperators appear starting from a situation with all defectors. Are such small mutations eliminated by the evolutionary dynamics, so that the original benchmark case is restored (case of transverse stability) or do they grow up thus causing an irreversible departure from the original benchmark case?

An answer to these questions requires the study of the local stability of the boundary equilibria, that is, the localization, in the complex plane, of the eigenvalues of the Jacobian matrix of (9.25) computed at the boundary steady states. This is not difficult in principle, as eigenvalues are always real because the Jacobian matrix of (9.25) is a triangular matrix along the invariant edges. This implies that we can only have nodes or saddle equilibria on the boundaries. However, the expressions of the eigenvalues are quite involved, and the stability conditions obtained are not easy to interpret.

So, in the following we prefer to follow a numerical and graphical 
method in order to obtain a global view of the dynamic properties of the dynamical system $(9.25){ }^{4}$

First of all, we consider the question of the existence of inner equilibria, that is, steady states characterized by the coexistence of cooperators and defectors. These are obtained solving the non-linear system

$$
\begin{gathered}
X(\alpha-\beta X)=H^{*}(X, s) \\
\pi_{c}^{*}(X, s)=\pi_{d}^{*}(X, s)
\end{gathered}
$$

with $0<\mathrm{s}<1$. The set of points on the plane $(X, s)$ that satisfy the first equation represent the locus of points that give a one-period stationary resource stock, that is, $X(t+1)=X(t) .{ }^{5}$ For each $s$ in the range $[0,1]$ the $X$ coordinates of these points can be computed by solving the equation (9.15), already analyzed in Section 3. So, this set of points may be formed by two branches, say $X_{1}(s)$ and $X_{2}(s)$, with $X_{2}(s)<X_{1}(s)$ for each $s$. Moreover, from the results of Section $3, X_{2}(s)$ is a decreasing function and $X_{1}(s)$ is increasing, so the branch $X_{1}(s)$ has a positive slope and the branch $X_{2}(s)$ has a negative slope in the plane $(X, s)$ (see Figures 9.4(a) and 9.5(a)). The

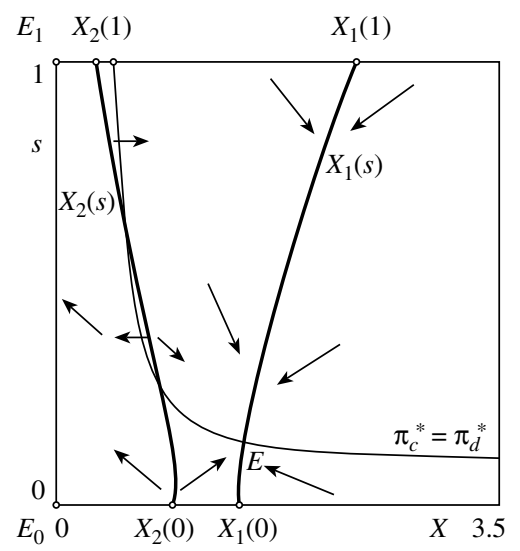

(a)

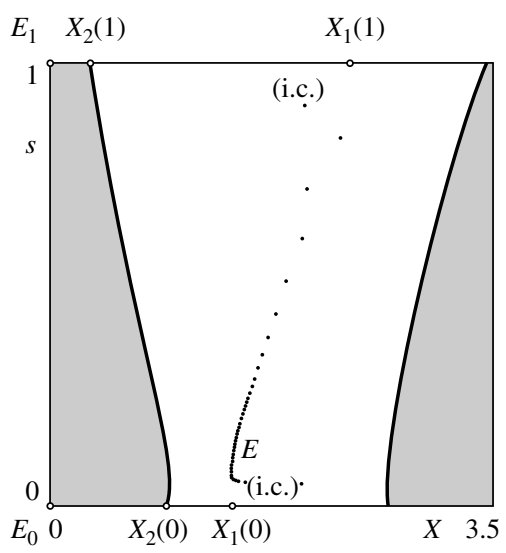

(b)

Notes:

(a) Obtained with the set of parameters $n=10, \alpha=3, \beta=1, a=1.05, b=0.3, \gamma_{c}=\gamma_{d}=1 \psi=0$, $\xi=0.01$. The arrows give a qualitative indication of the directions of one-step advancement of the discrete dynamical system (9.26). (b) For the same parameters as those used in (a) the basins of attraction are represented: the white region represents the basin of the inner equilibrium $E$, the grey region is the set of points that generate trajectories leading to extinction (that is, $X \leq 0)$. Two trajectories, starting from initial conditions $(2,2,0.9)$ and $(2$, $0.05)$, are also represented by sequences of black dots.

Figure 9.4 Numerical graphical representation of the equation (9.29) 


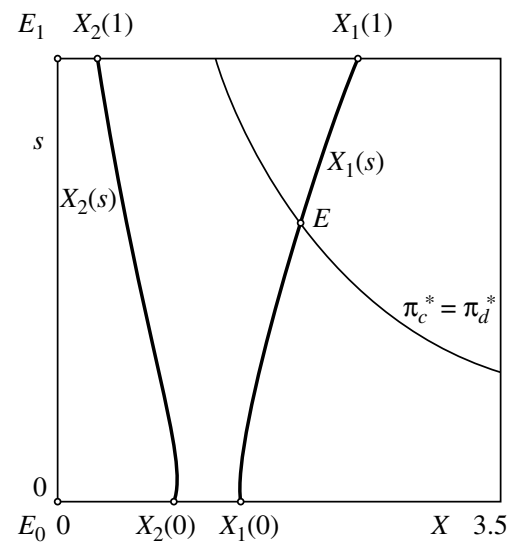

(a)

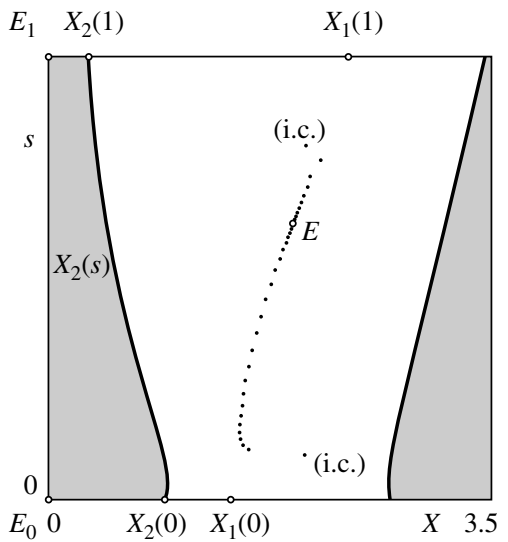

(b)

Figure 9.5 Numerical graphical representation of equation (9.29), with $\xi=0.05$

intersection of the branch $X_{1}(s)\left(X_{2}(s)\right)$ with the invariant edge $s=1$ gives the boundary equilibrium of $X$ coordinate $X_{1}(1)\left(X_{2}(1)\right)$, and the same holds for the intersections (if any) with the other invariant edge $s=0$. However, it may happen that the two branches intersect at $s=1$ but have no intersections with $s=0$, because they may merge for $s>0$ (see Figure 9.6(a)). If the condition (9.16) is satisfied for all $s \in[0,1]$, then the locus of points such that $X(t+1)=X(t)$ is only formed by the branch $X_{1}(s)$. The knowledge of these curves gives us the following information: starting from a given point $(X, s)$, a one-step iteration of (9.25) generates a new point $\left(X^{\prime}\right.$, $\left.s^{\prime}\right)=T$, rank-1 image of $(X, s)$ by $T$, with $X^{\prime}>X$ if $(X, s)$ is in the strip between the curves $X_{2}(s)$ and $X_{1}(s)$ (or between the axis $X=0$ and the curve $X_{1}(s)$ if only the branch $X_{1}(s)$ exists) and with $X^{\prime}<X$ if $(X, s)$ is on the left of the curves $X_{2}(s)$ (provided it exists) or on the right of the curve $X_{1}(s)$.

A similar reasoning can be applied to the set of points that satisfy the second equation (9.28), which represents the locus of points that give a oneperiod stationary population share, that is, $s(t+1)=s(t)$. A qualitative study of this curve is more difficult, due to the complicated expressions of $\pi_{c}^{*}(X, s)$ and $\pi_{d}^{*}(X, s)$. However, the numerical solution of the equation $\pi_{c}^{*}(X, s)=\pi_{d}^{*}(X, s)$ for different sets of parameters gives rise to decreasing curves in the plane $(X, s)$, as shown in Figures 9.4(a), 9.5(a) and 9.6(a). The points above the curve $\pi_{c}^{*}(X, s)=\pi_{d}^{*}(X, s)$ are points where $\pi_{c}^{*}(X, s)<\pi_{d}^{*}(X, s)$, hence the rank-1 images $\left(X^{\prime}, s^{\prime}\right)=T(X, s)$ of points $(X$, $s$ ) above the curve have $s^{\prime}<s$. Of course, the points below the curve are characterized by $\pi_{c}^{*}(X, s)>\pi_{d}^{*}(X, s)$, hence $\left(X^{\prime}, s^{\prime}\right)=T(X, s)$ are such that 


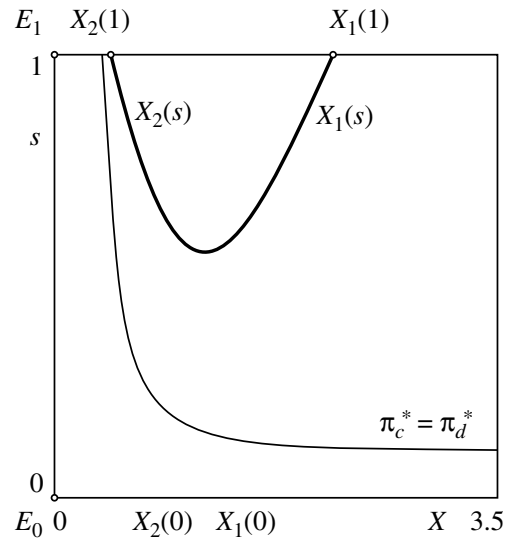

(a)

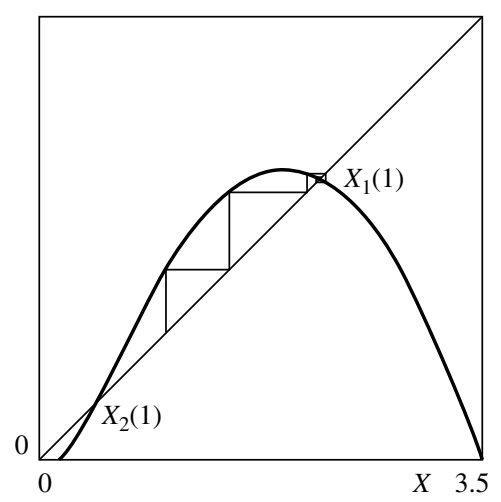

(b)

Notes:

(a) Parameters as for Figure 9.4a, except for $\alpha=1.2$. (b) (b) For the same parameters as those used in (a) the function $F(X)$ that governs the one dimensional dynamics along the invariant edge $s=1$ is represented, with a trajectory starting from $X=1$ and converging to the equilibrium $X_{1}(1)$.

Figure 9.6 Numerical graphical representation of equation (9.29), with $\alpha=1.2$

$s^{\prime}>s$. These arguments allow us to obtain a global qualitative picture of the dynamic behaviour of the dynamical system (9.25), as shown by the arrows represented in Figure 9.4a.

Any intersection between the curve $\pi_{c}^{*}(X, s)=\pi_{d}^{*}(X, s)$ and one of the curves $X_{i}(s), i=1,2$, represents a steady state of the dynamical system. All the steady states along the branch $X_{2}(s)$ cannot be stable, because all the points on that branch behave as repelling points along the $X$ direction. Instead, steady states located along the branch $X_{1}(s)$ are candidates to be stable equilibria.

We try to explain this point by some numerical experiments. In Figure 9.4(a), obtained with the set of parameters $n=10, \alpha=3, \beta=1, a=1.05, b=$ $0.3, \gamma_{c}=\gamma_{d}=1, \psi=0, \xi=0.01$, the two equilibria on $X_{2}(s)$ are unstable, whereas the equilibrium point $E=(1.48,0.15)$, located on $X_{1}(s)$ is stable. In Figure 9.4 b, the numerically computed basin of attraction of the stable equilibrium $E$ is represented by the white region, whereas the grey region represent the initial conditions leading to extinction of the resource. Two typical trajectories are also represented as sequences of black dots. From this picture the role of the curve $X_{2}(s)$ is quite evident: as in the one-dimensional model studied in Section 3 , the locus points $X_{2}(s)$ constitute the boundary that sep- 
arates the basin of attraction of the stable positive equilibrium from the basin of the trajectories leading to the extinction of the resource.

In the situation shown in Figure 9.4, the long-run evolution of the system leads to an equilibrium situation characterized by a small fraction of cooperators (only 15 per cent of the fishermen population). The number of cooperators at the stable equilibrium can be increased by varying some parameters, for example by increasing $\xi$, that is, imposing heavier sanctions, or by decreasing $a / b$, that is, lowering prices. Both these variations cause an upward movement of the curve $\pi_{c}^{*}(X, s)=\pi_{d}^{*}(X, s)$. For example, Figure 9.5 has been obtained by increasing the parameter $\xi$ from 0.01 to 0.05 . The consequence is that the new stable equilibrium is $E=(1.91,0.62)$, that is, 62 per cent of fishermen are cooperators and, consequently, a higher resource stock is present at equilibrium. If, starting from this situation, the parameter $\xi$ is further increased, or $a / b$ is decreased, the equilibrium $E$ moves upwards along the curve $X_{1}(s)$ until it merges with the boundary equilibrium $X_{1}(1)$ through a transcritical (or stability exchange) bifurcation. After this, the boundary equilibrium $X_{1}(1)$ becomes the unique stable steady state, where only cooperators exist. This means that prices are so low that it is not convenient to be defectors, even if very few defectors are present, that is, even if a defector appears (a mutation in the population composition), it is eliminated by the evolutionary mechanism.

It is also interesting to see what happens when prices are very high, as in the situation shown in Figure 9.6(a), where the same parameters as in Figure 9.4 have been used, except for the parameter $a=1.2$. In this case no positive boundary equilibria exist along the invariant boundary $s=0$ with all defectors, and two positive equilibria, $X_{2}(1)<X_{1}(1)$ exist along the edge $s=1$. In this situation all the initial conditions generate trajectories that approach the boundary $s=0$ and then converge to the unique stable equilibrium $E_{0}=(0,0)$. This means that prices are so high that defectors prevail, but their harvesting is so high that the resource is exhausted. However, if we constrain the system to start with $s=1$ (all cooperators) and sufficient initial resource stock, that is, $X(0)>X_{2}(1)$, then the system evolves towards the equilibrium $X_{1}(1)$, according to the one-dimensional dynamics shown in Figure 9.6(b). However, the equilibrium is not transversely stable. This implies that if just one defector appears, the endogenous evolutive dynamics will create more and more defectors, and the system will irreversibly evolve towards a situation with all defectors and extinction of the resource. 


\section{CONCLUSIONS}

In this chapter we have presented a model that constitutes an attempt to put together two opposite approaches to commercial fishing: cooperation (that is, harvesting decisions obtained through the maximization of overall profit, that lead to more conservative resource harvesting) and the noncooperative, or defective, attitude (that is, harvesting decisions obtained through the maximization of personal profit without any concern for social welfare). Oligopoly models based on one of these opposite attitudes, that is, all agents behaving as cooperators or all agents behaving as defectors, have recently been proposed by Szidarovszky and Okuguchi, 2000 and 1998 respectively (see also Bischi et al., 2004, for a comparison between the two cases). Instead, in the model proposed in this chapter we describe a common property resource exploitation with a population of agents switching between non-cooperative and cooperative behaviour according to an evolutive mechanism, known as replicator dynamics, based on the idea that the fraction of agents playing the strategy that earns higher payoffs will increase in the next period (as proposed in Sethi and Somanathan, 1996). As in the paper by Sethi and Somanathan, our model includes the possibility that cooperators impose sanctions to punish defectors. Indeed, without any sanction, the choice to be a defector is the dominant one (that is, the more profitable) and the evolutive process will consequently eliminate the cooperators. Instead, the presence of sanctions may eliminate defectors in the long run, or lead to a stable coexistence between cooperators and defectors.

The focus of our work is mainly methodological, as its primary goal is the set-up of the dynamic model: starting from a static oligopoly game, where each group chooses its harvesting according to the Nash equilibrium of the game, we realize that such an equilibrium depends on the available resource stock and the population share, and both these quantities evolve according to their dynamic equations. So, we first introduce the law of motion that governs the growth of the renewable resource and then we introduce the replicator dynamics that governs the time evolution of the population share between cooperators and defectors. This leads us to the study of a nonlinear two-dimensional discrete time dynamical system. The results given in this chapter constitute only a first step towards a full understanding of the dynamic behaviours of the model, as we have given only a numerical and graphical characterization of the equilibria and their stability.

We can summarize our results as follows. First, the presence of many cooperators always leads to a relatively high level of the resource stock, and hence wealth in the long run, whereas many defectors can cause a severe depletion of the resource, and an enlargement of the basin of attraction of steady states 
with extinction of the resource. Second, from the point of view of a regulator, cooperative behaviour can be supported not only by increasing the level of sanctions, or lowering prices, but it is also possible to reach steady states characterized by the presence of only cooperators which are transversely stable, that is, even if a defector enters the market this behaviour does not spread through the population. Otherwise, low sanctions and/or high prices could lead to steady states with only defectors, and the potential depletion (even extinction) of the resource. In this case, even if the system starts from an initial condition with only cooperators, non-cooperative behaviour will prevail if just one agent decides to defect. Even if a qualitative analysis of the model can be undertaken using the methods discussed in this chapter, a more complete analysis of the effects of the parameters on the kinds of long-run evolution, as well as on the transverse stability of corner equilibria, is under development.

Further enhancements of this model could also relax the assumption of perfect foresight about next-period fish stocks, replacing it by a weaker (and more realistic) assumption on expectations formations (for example, assuming that agents have adaptive expectations based on the available resource stock). Another interesting improvement of the model might be to assume sanctions that are not constant, but depend on some index of performance (from an ecologic and economic point of view) of the fishery system, so that the optimal kind of sanction is decided by methods of dynamic programming.

\section{APPENDIX}

Proof of proposition 1. The existence and uniqueness of the intersection between the reaction curves (9.9) trivially follows from the linearity of the reaction functions. The positivity of both the coordinates of the intersection point can be deduced from the fact that $h_{d}(0)<h_{c}^{-1}(0)$ and $h_{c}(0)<h_{d}^{-1}(0)$. In fact, the first inequality can be written as $a X / b\left(1+(1-s) X+2 \gamma_{d}\right)<a / b(1-s) n$, which is equivalent to $b X+2 \gamma_{d}>0$, the second inequality can be written as $X / 2\left(b s n X+\gamma_{c}\right)<1 / b s n$, which is equivalent to $b s n X+2 \gamma_{c}>0$. The explicit computation of the intersection point gives

$$
x_{d}^{*}(X, s)=a X \frac{b s n X+2 \gamma_{c}}{b^{2} \operatorname{sn}(n(1-s)+2) X^{2}+2 b\left(2 n s \gamma_{d}+\gamma_{c}+\gamma_{c} n(1-s)\right) X+4 \gamma_{c} \gamma_{d}}
$$

and

$$
x_{c}^{*}(X, s)=\frac{X a-b(1-s) n x_{d}^{*}(X, s)}{b s n X+\gamma_{c}}
$$

from which the properties stated in (i) and (ii) are quite evident. Moreover, $x_{d}^{*}$ is an increasing and concave functions with respect to $X>0$. The total harvesting at the Nash Equilibrium, $H^{*}(X, s)=n\left[s x_{c}^{*}(X, s)+(1-s) x_{d}^{*}(X, s)\right]$, is an increasing and concave function with respect to $X .^{6}$ 
For $\mathrm{X} \rightarrow+\infty$ we get

$$
\begin{gathered}
H^{*}(+\infty, s)=n a\left[\frac{1}{b n[(1-s) n+2]}+\frac{1-s}{b[(1-s) n+2]}\right]= \\
=\frac{a(1+n(1-s))}{b[(1-s) n+2]}=\frac{a}{b}\left(1-\frac{1}{2+(1-s) n}\right)
\end{gathered}
$$

Proof of proposition 2. Let us consider the function $Q(X)=X(\alpha-\beta X)-H^{*}(X)$, whose zeros are the equilibria of (9.14). Trivially it is $Q(0)=0$, so $X_{0}=0$ is an equilibrium.

In order to prove $(a)$ we notice that the condition (9.16) states that the slope at $X$ $=0$ of the function $H^{*}$ is less than the slope at $X=0$ of the parabola. This is equivalent to saying that $Q^{\prime}(0)>0$. Hence $Q(X)>0$ in a right neighbourhood of $X=0$, whereas $Q(\alpha / \beta)=-H^{*}(\alpha / \beta)<0$. So, at least one equilibrium exists in the range $(0$, $\alpha / \beta)$. The uniqueness of the equilibrium follows from the fact that $Q(X)$ is a unimodal function for $X>0$. In fact, $Q^{\prime}(X)=\alpha-2 \beta X-H^{* \prime}(X)$ vanishes in a unique point, because we have $Q^{\prime}(0)=\alpha-H^{* \prime}(X)>0$ from $(9.16), Q^{\prime}(\alpha / 2 \beta)=-H^{* \prime}(\alpha / 2 \beta)$ $<0$ and $H^{\prime}(X)$ is a positive, decreasing and convex function. ${ }^{7}$

To prove $(b)$ we notice that the first inequality in (9.17) states that at $X=0$ the curve $H^{*}(X)$ has a slope greater than that of the parabola, and the second inequality states that the upper bound (that is, the horizontal asymptote) of $H^{*}$ is lower than the vertex of the parabola. In fact, the first inequality implies $Q^{\prime}(0)<0$, hence $Q(X)<0$ in a right neighbourhood of $X=0$, whereas $Q(\alpha / 2 \beta)=\alpha^{2} / 4 \beta-H^{*}(\alpha / \beta)>0$ (being $H^{*}(X)<\frac{a}{b}(1-1 / 2+(1-s) n)$ for each $\left.X \geq 0\right)$ and $Q(\alpha / \beta)=-H^{*}(\alpha / \beta)<0$ again. So, at least two positive zeros of $Q(X)$ exist inside the intervals $(0, \alpha / 2 \beta)$ and $(\alpha / 2 \beta$, $\alpha / \beta)$ respectively. However, more than two zeros of $Q(X)$ cannot exist, because $Q(X)$ cannot have more than two critical points due to the convexity of $H^{\prime}(X)$.

Finally, the conditions (9.18), obtained by reversing the second inequality in (9.17), state that the slope of $H^{*}(X)$ at $X=0$ is greater than that of the parabola, and the upper bound of $H^{*}$ is above the vertex of the parabola, and this implies that no positive intersections can exist.

\section{NOTES}

We thank Laura Gardini for useful discussions and comments. The usual disclaimer applies. This work has been carried out under the auspices of the grant 'Le interazioni fra settore ittico e ambiente', financed by the Italian Ministry for Agriculture, and under the auspices of the national research project 'Dynamic Models in Economics and Finance: Evolution, Uncertainty and Forecasting', MIUR, Italy.

1. Since $n$ is finite, admissible values of $s$ should be discrete, that is, $s=k / n$ with $k=0,1, \ldots$, $n$. However, as usual in population dynamics and evolutionary games modelling, we abstract from this and allow $s$ to be a real number in the interval $[0,1]$, even if we shall consider $n s$ and $n(1-s)$ integers that sum to $n$, by assuming some approximation of $n s$ to the nearest integer.

2. A more general growth function $G(X)=X R(X)$, with $R(0)>1, R(X)>0$ for $0<X<K$ and $R(X)<0$ for $X>K, K>0$ being the carrying capacity, may be considered. However, the choice $R(X)=1+\alpha-\beta X$, known as logistic growth, is one of the simplest and most commonly used for qualitative analysis purposes.

3. This may be interpreted as a resource extinction due to overcrowding effects, a definitely unrealistic situation in the context we are considering. 
4. A more standard analytical study of the stability of the boundary equilibria, obtained with the help of software packages for symbolic algebraic manipulation, is in progress.

5. Of course, this does not mean that the dynamic system is in equilibrium, unless $s(t)$ is also stationary, that is, the second equation (9.28) is satisfied as well.

6. From (10) with (29) and (30) it is possible to prove (we tested the long expressions by a computer package) that $H^{\prime}(X)>0$ and $H^{\prime \prime}(X)<0$. Details of these expressions are available from the authors.

7. From (10) with (29) and (30) it is possible to prove that $H^{* \prime \prime}(X)<0$ and $H^{* \prime \prime \prime}(X)>0$. Details of these expressions are available from the authors.

\section{REFERENCES}

Bischi, G.I. and M. Kopel (2002), 'The role of competition, expectations and harvesting costs in commercial fishing', in T. Puu and I. Sushko (eds), Oligopoly Dynamics: Models \& Tools, Heidelberg: Springer Verlag, pp. 85-109.

Bischi, G.I., M. Kopel and F. Szidarovszky (2004), 'Competition and cooperation in multi-agent fisheries', forthcoming in Annals of Operation Research.

Clark, C.W. (1990), Mathematical Bioeconomics, 2nd edn, New York: Wiley John and Sons.

Gordon, H.S. (1954), 'The economic theory of a common property resource: the fishery', Journal of Political Economy, 62, 124 42.

Hardin, G. (1968), 'The tragedy of the commons', Science, 162, 1243-7.

Hofbauer, J. and K. Sigmund (1998), Evolutionary Games and Population Dynamics, Cambridge: Cambridge University Press.

Levhari, D. and L.J. Mirman (1982), 'The Great Fish War: An Example Using a Dynamic Cournot-Nash Solution', in L.J. Mirman and D.F. Spulber (eds), Essays in the Economics of Renewable Resources, Amsterdam: North-Holland, 243-58.

Mesterton-Gibbons, M. (1993), 'Game-theoretic resource modelling', Natural Resource Modeling, 7, 93-147.

Sethi, R. and E. Somanathan (1996), 'The evolution of social norms in common property resource use', The American Economic Review, 86, 766-88.

Szidarovszky, F. and K. Okuguchi (1998), 'An oligopoly model of commercial fishing', Seoul Journal of Economics, 11, 321-30.

Szidarovszky, F. and K. Okuguchi (2000), 'A dynamic model of international fishing', Seoul Journal of Economics, 13, 471-76.

Taylor, P. and L. Jonker (1978), 'Evolutionarily stable strategies and game dynamics', Mathematical Biosciences, 40, 145-56.

Vega-Redondo, F. (1996), Evolution, Games and Economic Behaviour, Oxford: Oxford University Press.

Weibull, J.W. (1995), Evolutionary Game Theory, Cambridge, MA: The MIT Press. 